\title{
ACEITAÇÃO DA TECNOLOGIA VDI NO AMBIENTE UNIVERSITÁRIO: O CASO DO ISCTE-IUL
}

\author{
João Machado e Bráulio Alturas \\ Instituto Universitário de Lisboa (ISCTE-IUL), ISTAR -IUL, \\ Instituto Universitário de Lisboa, Av. Forças Armadas 1649-026 Lisboa, Portugal
}

\begin{abstract}
RESUMO
O tema virtualização é uma realidade presente no quotidiano e em muitos dos serviços que são utilizados diariamente. O constante progresso das tecnologias de informação possibilitou um aumento significativo dos recursos de armazenamento e capacidade de processamento disponíveis dentro das organizações e universidades. Criou também a capacidade e oportunidade de otimizar os recursos existentes. Este artigo apresenta um caso de estudo que visa entender qual o nível de aceitação e conhecimento no âmbito da aplicação de VDI na comunidade do ISCTE-IUL. A adoção do VDI teve como finalidade principal a compatibilidade de aplicações, o reaproveitamento de sistemas, poupança a médio e longo prazo, a mobilidade, segurança, otimização de recursos e processos, gestão e controlo eficiente, permitindo melhorar a produtividade. Este artigo identifica a importância que os utilizadores dão a uma das preocupações do ISCTE-IUL, sustentabilidade. É de notar a falta de conhecimento sobre o tema, as suas vantagens, desvantagens e qual o impacto que poderá ter na organização. No entanto, os inquiridos acreditam tratar-se de uma inovação positiva para a comunidade.
\end{abstract}

\section{PALAVRAS-CHAVE}

VDI, Virtualização, Tecnologia, Centro de Dados

\section{INTRODUÇÃO}

Nos últimos anos temos vindo assistir a uma evolução tecnológica que dá passos largos e, de certa forma, quase que somos forçados acompanhar este ritmo. Um dos alicerces e pensamento em a ter em conta para quem gere uma organização deverá ser evitar desperdícios, refletindo-se em maus investimentos em equipamentos informáticos.

O processo de instalação atual de uma máquina é moroso e exige a pré-alocação de pelo menos um recurso humano técnico para o fazer. Desde a instalação do SO, aplicações solicitadas para determinado projeto/área, licenciamentos e outras configurações necessárias tornam tudo muito complexo e fechado.

A virtualização dos Desktops veio permitir ultrapassar grande parte dos problemas do dia a dia das equipas das Tecnologias de Informação e oferecer soluções rápidas e ajustadas às necessidades dos próprios utilizadores. Torna o processo rápido, eficiente e com baixo risco de incidentes desde a criação da imagem até à entrega da mesma.

Um dos grandes benefícios da virtualização é a sua mobilidade e o acesso à máquina virtual (VM) pode ser feito através de qualquer dispositivo com rede (dentro e fora da rede empresarial), assim como podem ser definidos diferentes perfis de segurança e isolar áreas críticas para a instituição.

O desejo de investigar e permitir otimizar os processos e os workflows utilizados na área das tecnologias de informação são uma das partes mais importantes, pois as próprias instituições muitas vezes desconhecem os potenciais de otimização de produtividade. Disponibilizar aos utilizadores postos de trabalho que estejam sempre acessíveis a partir de qualquer localização, de forma segura e a partir de diferentes dispositivos, conferindo-lhes uma maior mobilidade no decurso das suas atividades, fundamentais para grupos como alunos, docentes e funcionários.

Este estudo visa responder à questão de investigação: "Qual o nível de aceitação e conhecimento no âmbito da aplicação de VDI na comunidade do ISCTE-IUL".

Foram definidos dois objetivos para responder a esta questão de investigação:

1. Analisar as caraterísticas, configurações e definições para que o VDI, quando implementado, 
permita uma melhoria constante na utilização desta tecnologia.

2. Entender qual o nível de aceitação e conhecimento no âmbito da aplicação de VDI na comunidade do ISCTE-IUL através da realização de um inquérito por questionário.

\section{REVISÃO DE LITERATURA}

\subsection{Virtualização}

A virtualização é a técnica que permite particionar um único sistema computacional em vários outros denominados de máquinas virtuais. Cada máquina virtual oferece um ambiente completo muito similar a uma máquina física. Com isso, cada máquina virtual pode ter seu próprio sistema operacional, aplicativos e serviços de rede" (Carissimi 2008) (Almeida 2011).

Sahoo, Mohapatra e Lath referem que "O objetivo do ambiente de computação virtual é melhorar a utilização de recursos, fornecendo uma plataforma operacional integrada unificada para utilizadores e aplicativos baseados na agregação de recursos heterogéneos e autónomos" (Sahoo, Mohapatra e Lath 2010).

Através das definições de Carrisimi, Sahoo, Mohapatra e Lath permite-nos chegar ao entendimento que este conceito de virtualização é uma poderosíssima ferramenta, com capacidade de abstração das aplicações e todos os seus componentes subjacentes, do próprio hardware que as suporta e representam assim recursos de uma forma lógica. Tendo mais potencial para que esta representação possa ser bastante diferente da sua representação física. Abre todo um novo mundo de possibilidades de gestão e operacionalidade que pode cruzar tecnologias e fabricantes, criando uma camada de heterogeneidade.

O hipervisor (Figura 1) conhecido como gestor de máquinas virtuais, é um processo que cria e executa VM. Um hipervisor permite que um computador host suporte várias VM convidadas compartilhando de forma virtual os seus recursos, tais como: memória, processamento, rede entre outros (VMware 2019).

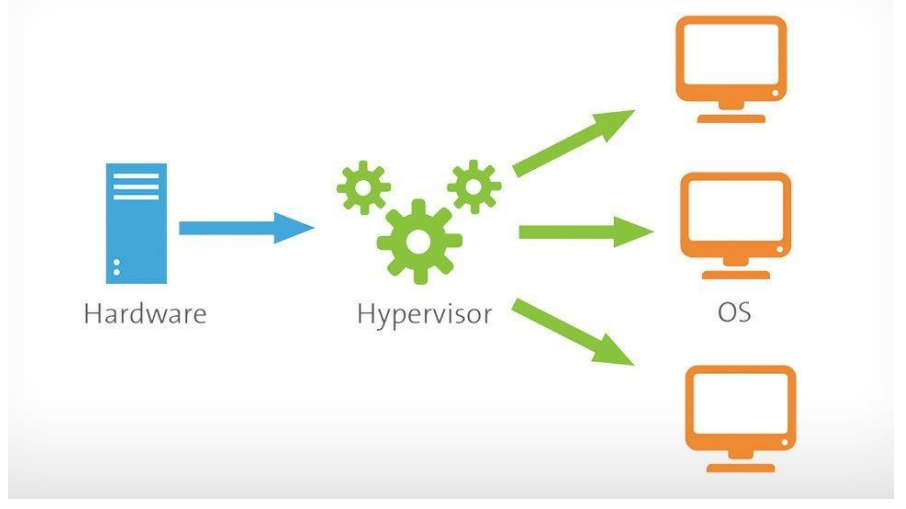

Figura 1. Exemplo de uma arquitetura de um hipervisor ${ }^{1}$

Existem duas tipologias principais de arquitetura de virtualização, as de tipo um e tipo dois (Figura 2) (VMware 2007).

\footnotetext{
${ }^{1}$ Imagem retirada do site: https://www.vmware.com/topics/glossary/content/hypervisor
} 


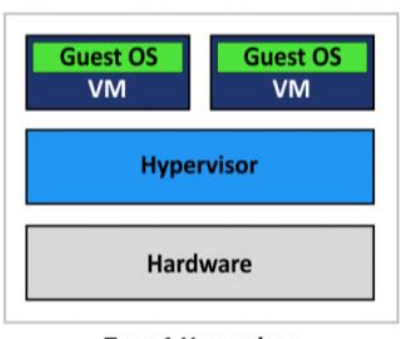

Type 1 Hypervisor

(Bare-Metal Architecture)

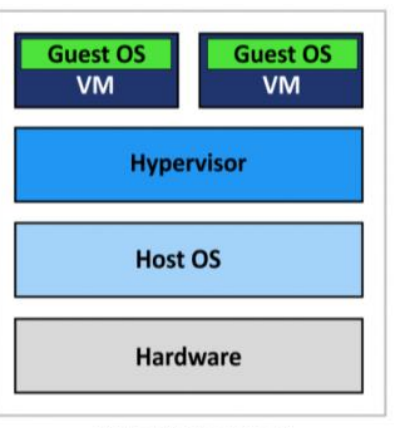

Type 2 Hypervisor

(Hosted Architecture)

Figura 2. Tipos de arquiteturas principais de hipervisores ${ }^{2}$

Virtual Machine Manager (VMM) tipo Bare-metal é o termo dado à execução de software diretamente sobre o hardware, sem nenhuma outra camada de software por cima. É um hipervisor criado especificamente para instalação direta sobre um servidor físico, tendo o controlo total sobre os recursos de hardware. É mais eficiente que as arquiteturas do tipo dois e pode particionar o próprio hardware para atingir maior eficiência.

Como exemplos de soluções tipo um, que utilizam esse tipo de hipervisor, temos o VMware ESXi, o Xen e o KVM (Kernel-based Virtual Machine) que são Kernels Linux modificados para trabalhar como hipervisor.

No tipo Hosted Architecture, o hipervisor é executado diretamente sobre um sistema operativo normal, chamado de hóspede. Logo, nesta situação o hipervisor tem controlo limitado sobre o hardware pois funciona como se fosse uma aplicação.

Como exemplos de soluções tipo dois, que utilizam esse tipo de hipervisor, temos o VMware Player/Fusion/Workstation, Parallels Desktop/Workstation, VirtualBox, entre outros. Esta solução tende a ser muito utilizada, pois não requer que o sistema operativo nativo seja substituído.

A virtualização assenta sobre um modelo (Figura 3) de sete camadas de tecnologias diferentes, sendo que cada uma delas representa parte de um ambiente de computação (Kusnetsky 2011).

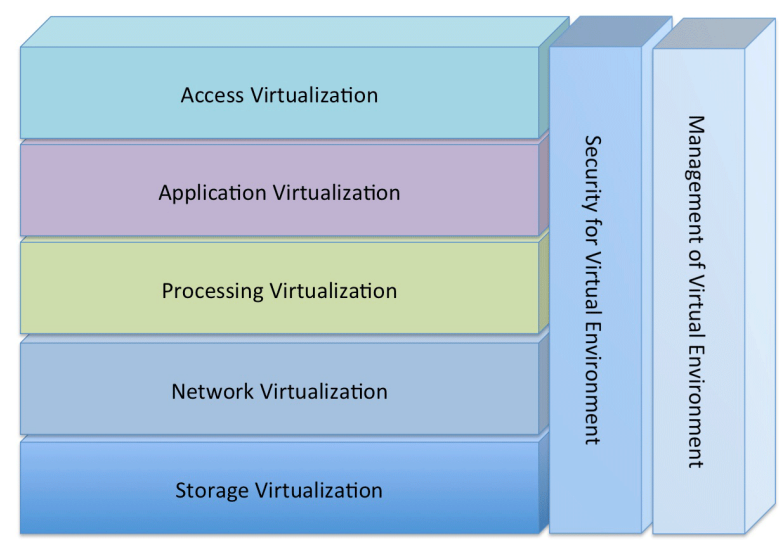

Figura 3. Modelos de Virtualização por camadas ${ }^{3}$

A VMware continua a liderar o mercado nesta área, mas a Microsoft está cada vez mais perto. A Citrix encontrou para já a sua posição confortável, mas continua a desenvolver soluções muito interessantes e a ter em conta (Gartner 2016).

Existem várias técnicas para implementar a virtualização: a Técnica da Virtualização Total e a Paravirtualização. A técnica da Virtualização Total aprovisiona uma completa simulação da subcamada de hardware para os sistemas convidados, como se de facto houvesse uma máquina física inteiramente à

\footnotetext{
${ }^{2}$ Imagem retirada do site: https://www.nakivo.com/blog/hyper-v-virtualbox-one-choose-infrastructure/

${ }^{3}$ Imagem retirada do site:

https://virtualizationreview.com/articles/2014/10/14/ /media/ECG/virtualizationreview/Images/2014/10/1014vrm_KusVirtLayersfig ure.ashx
} 
disposição. O VVM disponibiliza também a cada VM uma BIOS (Basic Input Output System) virtual, gestão de memória virtual e até dispositivos virtuais.

Existem duas formas de utilização desta técnica, sendo uma delas através da assistência por software e a outra através da assistência por hardware (VMware 2007):

- Assistido por software (BT - Binary Translation). Recorrendo a tradução binária, depende completamente das traduções binárias para conseguir intercetar e virtualizar a execução de conjuntos de instruções sensíveis e não virtualizáveis. Emula o hardware utilizando os conjuntos de instruções do software. Alguns dos softwares que utilizam esta técnica são: o Virtual Box, VMware Workstation, VMware Server entre outros (VMware 2007).

- Assistido por Hardware (VT - Virtual Technology). A virtualização total assistida por hardware elimina a tradução binária e interrompe diretamente o hardware utilizando a tecnologia de virtualização que foi integrada nos processadores x86, desde 2005 (Intel VT-x e AMD-V). As instruções dos sistemas operativos hospedes podem permitir que num contexto virtual executem instruções privilegiadas diretamente no processador, mesmo este estando virtualizado (Biswas \& Islam 2009) (Intel 2010) (AMD 2012). Alguns dos softwares que utilizam esta técnica são o VMware ESXI / ESX, KVM, Hyper-V, Xen, entre outros.

As principais vantagens destas duas técnicas de virtualização são a ausência da necessidade de alterações nos sistemas convidados, para que estes suportem a virtualização, pois a sua estrutura de hardware está totalmente virtualizada. Também oferece uma camada de segurança e melhor isolamento para as próprias VM.

Esta abordagem também pode ter algumas limitações consideráveis, uma delas é o risco de existirem pedidos de o sistema convidado não serem totalmente correspondidos e atendidos da maneira esperada. Um exemplo disso, um recurso de hardware que não possa ser acedido por falta de determinado driver no seu sistema de virtualização (VMware 2019).

Paravirtualização: Ao contrário da técnica de virtualização total, a Paravirtualização obriga à modificação do kernel do sistema operativo convidado com o propósito de substituir as instruções não virtualizáveis (hypercalls), que comunicam diretamente com o VMM. O hipervisor é instalado num servidor físico (host) e o sistema operativo hospede é instalado no seu ambiente (Williams 2007).

A sua principal vantagem é o seu maior desempenho, pois o VMM não é sobrecarregado com a tradução binária de instruções sensíveis. Elimina também a necessidade da dependência dos mecanismos trap do cpu, eliminando a necessidade de capturar e emular a maioria das instruções.

Esta abordagem também pode ter algumas limitações consideráveis, um exemplo disso é a necessidade da adaptação / modificação prévia do sistema operativo hóspede.

\subsection{Virtualização de Desktops e Infraestruturas (VDI)}

A virtualização de Desktops consiste no acesso feito por um utilizador, a uma determinada pool de máquinas virtuais (VM) ou simplesmente a uma VM dedicada, através de um terminal de acesso. Estes terminais podem ser um computador, um smartphone entre outros (Figura 4).

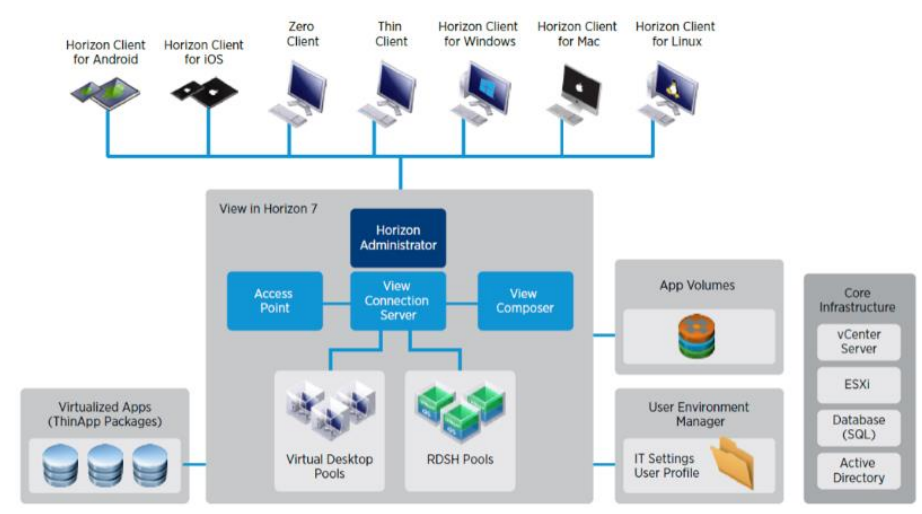

Figura 4. Representação dos acessos dos terminais a uma infraestrutura VDI ${ }^{4}$

\footnotetext{
${ }^{4}$ Imagem retirada do site: https://blogs.vmware.com/euc/2017/04/vmware-horizon-7-view-reviewers-guide-complete.html
} 
A experiência de utilização proporcionada aos utilizadores desta tecnologia é semelhante à de um computador convencional/normal. O seu tipo de arquitetura cliente - servidor transmite toda a necessidade de processamento para o servidor, permitindo assim que o próprio cliente de acesso seja um terminal simples, sem disco rígido por exemplo. A única responsabilidade do terminal é encaminhar os inputs e outputs para o servidor remoto, cumprindo com as instruções solicitadas pelos seus utilizadores.

Este tipo de equipamento (ThinClient) tem um consumo de energia muito reduzido, não disponibiliza uma grande performance pois não é esse o seu propósito. Praticamente não têm componentes mecânicos, por sua vez o seu custo também é muito inferior quando, comparado a um computador convencional. O seu ciclo de vida também é superior e oferece maior segurança, uma vez que toda a informação é centralizada no centro de dados (Ahmed e Moukali 2014).

Estes sistemas possuem um conjunto de workloads e automatismo que permitem gerar uma ou mais VM em alguns segundos, com um sistema operativo já pré-preparado e configurado à medida, incluir files shares com os dados de cada utilizador assim como disponibilizar aplicações no momento. Estas VM podem ser destruídas ao final de cada utilização e o sistema pode ser configurado para preservar todos os dados do utilizador e disponibilizá-los no seu próximo acesso (VMware 2017).

Esta tecnologia oferece diversas vantagens, mas também tem as suas desvantagens que podem representar novos desafios para as instituições que pretendem implementar esta solução.

As vantagens principais desta tecnologia são: a estabilidade; alta disponibilidade; segurança; mobilidade e a redução de custos a médio prazo (VMware 2015) (Pires 2017) (Ribeiro 2016).

As principais desvantagens são: o investimento inicial elevado; maior complexidade na interface de gestão; elevada dependência da infraestrutura e a garantir a performance (Pires 2017) (Ribeiro 2016) (Shabaitah 2014). E para além destas vantagens A utilização do VDI é uma maneira de reduzir a pegada de carbono no nosso planeta e economizar nos custos de energia (Chrobak 2014).

\section{METODOLOGIA}

Após a abordagem teórica à tecnologia de virtualização e à virtualização de desktops de infraestruturas (VDI) será estudado como a implementação VDI é aceite ou não pelos usuários da comunidade ISCTE-IUL.

Como mencionado anteriormente a questão de investigação é "Qual o nível de aceitação e conhecimento no âmbito da aplicação de VDI na comunidade do ISCTE-IUL".

O método de pesquisa adotado foi o caso de estudo.

A abordagem metodológica foi baseada na aplicação de questionários a três grupos distintos da universidade ISCTE - Instituto Universitário de Lisboa, sendo o primeiro grupo os 370 Docentes, o segundo grupo os 270 Funcionários e por fim, o terceiro grupo os 9000 Estudantes.

A construção destes questionários foi desenvolvida numa primeira fase com base em entrevistas de resposta aberta a cinco elementos chave (Key Users) de cada um dos grupos (Docentes, Funcionários e Estudantes) pois foi importante perceber quais as suas dificuldades, dúvidas, sugestões entre outras. Os dados foram recolhidos no período de 20 de março a 20 de abril de 2019.

Numa segunda fase, desenvolveu-se um outro questionário com base na revisão da literatura e nas entrevistas da fase anterior. Foram criadas questões para entender e analisar a perceção, conhecimento e preocupação de cada utilizador na utilização do VDI, utilizando escalas do tipo Likert de cinco pontos, e aplicadas a uma amostra de 121 indivíduos das três categorias principais dentro do ISCTE-IUL: 60 Alunos, 31 docentes e 30 funcionários. Foi também disponibilizado um exemplo de uma máquina virtual no âmbito desta investigação, com um sistema operativo e um conjunto de aplicações padrão. Os dados recolhidos no período de 01 de maio a 20 de maio de 2019.

\section{RESULTADOS}

Foram analisadas as caraterísticas, configurações e definições do VDI que permitam uma melhoria constante na utilização desta tecnologia na organização foi também elaborado um conjunto de questões sobre: reutilização de recursos, cibersegurança, relevância no VDI, formação e conhecimento sobre esta tecnologia. 
Os inquiridos (55\%) têm a alguma perceção da reutilização de recursos físicos, aquando a utilização do VDI, como por exemplo a utilização de computadores mais antigos.

A maioria dos inquiridos respondeu que o novo conceito VDI gerou alguma (46\%) ou muita alteração (39\%) em ultrapassar a complexidade e inflexibilidade dos sistemas atuais. Com base nos resultados depreende-se que existe uma tendência para a aceitação do novo conceito VDI em melhorar, por exemplo, tempos de resposta a novos pedidos/alterações no computador.

Tanto para os alunos (46,67\%), como para os docentes $(41,94 \%)$ e para os funcionários $(33,33 \%)$ a sustentabilidade é um tema razoável em que não é dada nem muita nem pouca importância. O que revela que nos dias de hoje a sustentabilidade ainda é um tema a ser trabalhado pelo ISCTE-IUL. De salientar ainda que os alunos são os que consideram o sistema VDI mais amigo do ambiente, consideram que é indispensável para a poupança de energia e recursos.

Os inquiridos mostram-se bastante preocupados com o tema da cibersegurança. Utilizando uma escala do tipo Likert de cinco pontos (dispensável, pouco importante, razoavelmente importante, muito importante e indispensável), verificou-se que este tema é muito importante ou indispensável para 54\% dos inquiridos, sendo os alunos os que demonstram mais preocupação sobre este tema (indispensável 36,67\%) comparativamente às outras categorias profissionais (funcionários $26,67 \%$ e docentes $25,81 \%$ ). Dentro do mesmo tema foi também avaliada a preocupação sobre possíveis ataques ou incidentes de segurança no geral das empresas fora da organização e apenas uma minoria respondeu ser um tema dispensável ou pouco preocupante (9\%). Constatou-se que para as categorias alunos e docentes é de importância razoável trabalhar numa máquina atualizada, assim como as respetivas aplicações. Já para os funcionários uma máquina atualizada é indispensável $(33,33 \%)$ para a boa execução das suas tarefas.

A preservação de dados é a característica mais importante para os inquiridos aquando a utilização de um computador físico ou virtual, seguida do nível de segurança e as menos importantes são o bloqueio de publicidades e tracking e o Single Sign On.

A maioria dos inquiridos $(72,73 \%)$ nunca frequentou formação relacionada com os VDI, destaca-se a categoria de alunos em que $45 \%$ responde não ter qualquer tipo de conhecimento acerca do tema. Já no caso dos docentes e funcionários esse valor é bastante inferior com 25,81\% para os docentes e $16,67 \%$ para os funcionários.

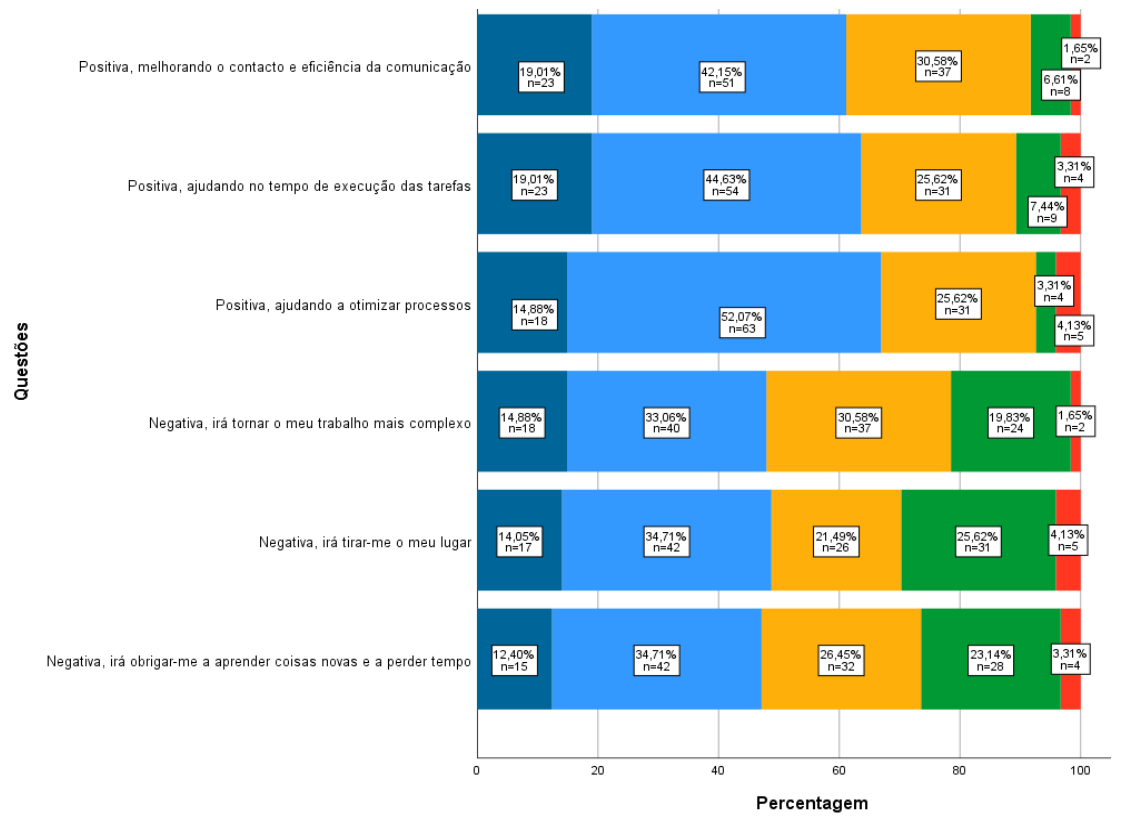

Figura 5. Perceção da mudança no local de trabalho através da implementação do VDI

Relativamente à utilização da tecnologia em estudo por parte dos inquiridos, pouco menos de metade (44\%) já utilizou esta ferramenta.

A Figura 5 avalia a opinião dos inquiridos relativamente à forma como o VDI pode mudar, positivamente ou negativamente, a sua forma de trabalhar. 
De forma positiva a otimização de processos é vista como a maior vantagem $(66,95 \%)$ sendo que o tempo de execução das tarefas e a eficiência da comunicação também têm grande destaque. De forma negativa os inquiridos vêm como ameaça ao seu posto de trabalho $(48,76 \%)$ a implementação do VDI, sendo que a complexidade do trabalho e obrigação de aprendizagem de novos procedimentos e a consideração como uma perda de tempo tem um elevado número de respostas de concordo parcialmente e totalmente o que poderá criar alguma resistência à mudança quando a implementação do VDI no posto de trabalho.

\section{CONCLUSÃO}

Este estudo pretende responder à questão inicial de investigação "Qual o nível de aceitação e conhecimento no âmbito da aplicação de VDI na comunidade do ISCTE-IUL”.

Os objetivos propostos foram parcialmente alcançados:

1. A análise das caraterísticas, configurações e definições para que o VDI permita uma melhoria constante na utilização desta tecnologia num ambiente universitário. só seria totalmente possível com o sistema VDI já implementado no ISCTE-IUL. Tendo sido apenas criado um ambiente de teste para a realização desta investigação. O procedimento atual passa pela criação de uma imagem padrão, em que a revisão só é realizada no ano letivo seguinte apesar de existirem pequenas atualizações ao longo do ano. Sempre que exista necessidade de proceder a uma alteração na imagem base, a entrega de uma nova versão é morosa o que poderá não responder atempadamente às necessidades dos utilizadores. O VDI permite ultrapassar este tipo de complexidade e inflexibilidade por parte do modelo atual, através da entrega de pacotes de containers de aplicações ou mesmo individualizadas. Podem ser geradas várias máquinas virtuais com imagens base diferentes, criando assim uma capacidade de resposta aos pedidos realizados ao longo do ano letivo pelos utilizadores.

2. Relativamente ao segundo objetivo foi cumprido e conclui-se que a comunidade ISCTE-IUL não se sente preparada e não possui ainda conhecimento suficiente acerca do conceito do VDI. Por parte dos funcionários poderá existir alguma resistência à mudança do seu método de trabalho, por desconhecimento das vantagens e desvantagens desta tecnologia. Aquando a implementação do sistema VDI deverão ser realizadas ações de formação introdutórias para dar conhecimento do modo de funcionamento, impacto e qual a finalidade para todas as categorias profissionais.

Os resultados apresentados pelos inquiridos do ISCTE-IUL vão ao encontro da não entrada em produção em grande escala do VDI, resumindo-se a um grupo restrito de pessoas que testaram a solução ainda em modo de testes, em pré-implementação. Os resultados revelam a pouca ou inexistente utilização desta tecnologia, desta forma também é complexo para os inquiridos avaliar de que forma o VDI poderá influenciar a performance no local de trabalho (se de forma positiva ou negativa e porquê) e tempos de entrega de novas aplicações e sistemas operativos.

Contudo existe a perspetiva de crescimento a curto prazo acompanhando a tendência global ao nível de sistemas de informação.

As principais limitações deste estudo foram o desconhecimento generalizado da solução VDI, refletindo-se nos resultados obtidos através dos questionários. Seria importante ter a solução já implementada e enraizada na instituição, acessível a todos para que pudesse ser testada com mais tempo sem ter de se recorrer a uma alternativa cloud. Assim tornou-se menos claro de entender qual o impacto final no utilizador.

A evolução tecnologia é uma tendência crescente e seria interessante de futuro investigar e testar as três principais soluções atuais no mercado, em vez de apenas uma, pois permitiria comparar mais dados. A realização de nova investigação após a implementação final na comunidade ISCTE-IUL, de forma a analisar o impacto na organização. Entender também quais as áreas científicas com maior necessidade de formação/conhecimento, pois o VDI é uma ferramenta transversal a qualquer utilizador. Para finalizar, a utilização de placas gráficas (virtual GPU- Graphics processing unit) na solução VDI por forma analisar o comportamento e performance das mesmas, quando estas partilham os seus recursos pelas várias máquinas virtuais. 


\section{REFERÊNCIAS}

Ahmed, S. \& Moukali, K. 2014. "An Efficient Implementation of Thin Client Technology for E-learning in the Jazan University". International Conference on Web and Open Access to Learning, ICWOAL.

Almeida, A. 2011. "Virtualização", Universidade do Porto.

AMD 2012. “AMD-V Nested Paging” Jun. [Online-2019]. Disponível: http://developer.amd.com/wordpress/media/12/10/NPT-WP-1\%201-final-TM.pdf

Biswas, K. \& Islam, A. Md. 2009. "Hardware Virtualization Support In INTEL, AMD And IBM Power Processors". International Journal of Computer Science and Information Security, 4(1).

Carissimi, A. 2008. "Virtualização: da teoria a soluções", $26^{\circ}$ Simpósio Brasileiro de Redes de Computadores e Sistemas Distribuídos, pp.173-207.

Chrobak, P. 2014. "Implementation of Virtual Desktop Infrastructure in academic laboratories". Federated Conference on Computer Science and Information Systems, pp. 1139-1146.

Gartner 2016. "Magic Quadrant for x86 Server Virtualization Infrastructure”, Gartner.

Intel 2010. "Server, Enabling Intel® Virtualization Technology Features and Benefits", White Paper, Intel.

Kusnetzky, D. 2011. "Virtualization: A Manager's Guide”. O’Reilly.

Pires, A. J. A. C. P. 2017. "Implementação de um sistema de virtualização de postos de trabalho na faculdade de ciências e tecnologia". Dissertação de mestrado. UALg -Universidade do Algarve.

Ribeiro, B. M. R. 2016. "Virtualização de recursos de TI: Estudo de Caso do ISQ". Bachelor Thesis. ISQ - Instituto de Soldadura e Qualidade

Sahoo, J., Mohapatra, S. \& Lath,R. 2010. "Virtualization: A survey on concepts, taxonomy and associated security issues", 2nd International Conference on Computer and Network Technology. ICCNT 2010, pp. 222-226.

Shabaitah, A. R. 2014. "Server-Based Desktop Virtualization". Thesis. Rochester Institute of Technology.

VMware 2007. "Understanding Full Virtualization, Paravirtualization, and Hardware Assist", Memory, White paper, 17.

VMware 2015. "VDI-A New Desktop Strategy" 2015 [Online-2019]. Disponível: https://www.vmware.com/pdf/vdi_strategy.pdf

VMware 2017. "Horizon 7 Instant-Clone Desktop and RDSH Servers" [Online-2019]. Disponível: https://www.vmware.com/content/dam/digitalmarketing/vmware/en/pdf/techpaper/vmware-horizon-view-instantclone-technology.pdf

VMware 2019. "Hypervisor". [Online-2019]. Disponível: https://www.vmware.com/topics/glossary/content/hypervisor

Williams, D. E. 2007. "Virtualization with Xen (tm): Including XenEnterprise, XenServer, and XenExpress". Elsevier. 